Wouters, C.; Wijnhoven, T.; De Wit, K.; Vanwynsberghe, F.; Deconinck, G., "Reliability analysis of grid concepts," in IEEE Power and Energy Society General Meeting (PES), Vancouver, BC, Canada, 21-25 July 2013, 5 p.

doi: 10.1109/PESMG.2013.6672096

URL: $\underline{\text { http://ieeexplore.ieee.org/stamp/stamp.jsp?tp=\&arnumber=6672096\&isnu }}$ $\underline{\mathrm{mber}}=6672065$

(c) 2013 IEEE. Personal use of this material is permitted. Permission from IEEE must be obtained for all other users, including reprinting/ republishing this material for advertising or promotional purposes, creating new collective works for resale or redistribution to servers or lists, or reuse of any copyrighted components of this work in other works.

(below follows the accepted version) 


\title{
Reliability analysis of grid concepts
}

\author{
Carmen Wouters*, Thomas Wijnhoven*, Kris De Wit ${ }^{\dagger}$, Frank Vanwynsberghe* ${ }^{\dagger}$ and Geert Deconinck* \\ * ELECTA, Department of Electrical Engineering, KU Leuven, Belgium \\ $\dagger$ Electrical Power Supply, BASF Antwerp, Belgium
}

\begin{abstract}
Traditional techniques that evaluate the reliability of electrical grids lack the capability of providing an objective basis to assess the reliability of industrial grids. Therefore, there is an increasing demand towards probabilistic techniques that are able to create a more solid basis regarding future grid investments and to justify implementation decisions made in the grid. In this paper, the probabilistic method of the discrete absorbing Markov chain is proposed together with the matrix multiplication method to gain insight in both the reliability and the average cost of production loss due to faults in an industrial electrical grid. The merits of this technique are illustrated through two case studies in which parts of the electrical grid of the chemical production site of BASF Antwerp are simulated. From these case studies, both the weakest parts of the grid are identified as well as the average cost of production loss after one year.
\end{abstract}

Index Terms-industrial power system reliability, Markov processes, matrix multiplication

\section{INTRODUCTION}

The major role of an industrial electrical grid is to provide its consumers continuously and in an economical manner with sufficient energy to sustain their production [1], [2]. As such, the electricity supply and distribution must be secured at all times to limit the possible costs of production loss of the consumers as well as to limit damage towards installations due to the occurrence of a fault in the grid [3]. Therefore, the reliability, as a part of the dependability, of the electrical grid must be high. To achieve the desired reliability level, grid designers and operators used to employ their own practical criteria like for example LOLE, Loss Of Load Expectation [2]. Today, several probabilistic models are in use to determine the reliability level of an electrical grid in a more objective manner [4]. These models take several aspects into account like for example the failure rates of the different grid components [2].

This paper describes the work done in [5] and is organised as follows: In Section II, literature is consulted to find the most suitable probabilistic model [6]-[9]. The chosen model must satisfy several requirements from the industrial company. Furthermore, the chosen method of the discrete absorbing Markov chain is described as well as the way it is implemented. With this model, two case studies are investigated in Section III. Finally, the results of the case studies and the economic analysis are explained and interpreted.

T. Wijnhoven has a Ph. D. fellowship of the Research Foundation - Flanders (FWO) and wishes to acknowledge the financial support of the FWO.

E-mail contact: thomas.wijnhoven@esat.kuleuven.be

\section{Probabilistic METHOD}

The probabilistic modelling technique must satisfy the industrial requirements. Literature provides different methods to consider [6], [7], [9], [10]. Apart from the reliability, also the economical aspect of the average damage cost, resulting from failures, must be demonstrated for different grid configurations. For complex electrical industrial networks, like the electrical grid of the chemical production site of BASF Antwerp, two probabilistic modelling techniques are most suitable: Event Tree Analysis and Markov Method. In this section the choice of the probabilistic model is clarified as well as its method of operation. In a next step, the proposed method is introduced for two case studies to evaluate the electrical grid of BASF Antwerp.

\section{A. Model requirements}

Major industrial electrical consumers demand a probabilistic reliability model that meets following requirements:

- The model should quantify the effects of electrical component failures on the reliability level of the grid.

- The model must include a time aspect and must be able to take maintenance and repair into account.

- The model should be easily expandable to other grid configurations and voltage levels.

\section{B. Model choice}

After a first assessment of the modelling techniques found in literature, the Event Tree Analysis and the Markov Method appear to be the most suitable and flexible probabilistic modelling techniques. Although the Markov Method becomes fairly complex for larger networks, like the grid of BASF Antwerp, this method has a major strength compared to the Event Tree Analysis. The Markov Method is able to integrate time into the simulations. This is an important aspect, as this feature enables to obtain the probabilities of the defined states after a chosen time period [6], [8], [11]. As the Markov Method satisfies all requirements, this model is proposed as the most suitable method for further implementation. The investigated grid will move in between different defined states and will make stepwise transitions between those states [6][9]. The transitions between the different states of the grid can occur based on fixed determined fail- and repair rates. As in this case the investigated grid moves in between different defined states, a discrete Markov model is chosen: the Markov chain. A Markov chain is usually simulated using a discrete 


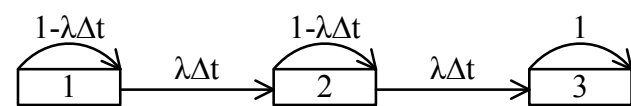

Fig. 1. A state space diagram of a DAMC

time step, $\Delta \mathrm{t}$, to reduce calculation time. In this way, the proposed model becomes a finite discrete Markov chain.

The probabilities of the defined final states of the grid must be found for a chosen simulation period. In a first evaluation of the grid, repair is omitted (see case study I and case study II). In this way, the final states become so called 'absorbing' states as the grid remains in these states with a probability equal to one once they are attained [6], [8], [11]. The model used for further simulations thus becomes a discrete absorbing Markov chain (DAMC).

\section{Discrete Absorbing Markov Chains}

In this DAMC model, every grid component has a constant failure rate, $\lambda$, and repair rate, $\mu$, as the grid components are assumed to be in the period of their useful lifetime [8], [10]. As such, every component follows an exponential failure law. Here, the failure rate and repair rate are the reversed values of respectively the Mean Time To Failure (MTTF) and the Mean Time To Repair (MTTR). With the exponential failure law, the failure rate of the major grid components within a small time step can be expressed as follows [8], [12].

$$
1-e^{-\lambda \Delta t} \approx \lambda \Delta \mathrm{t}
$$

As this time step is kept constant throughout the simulation, the model becomes homogeneous in time and can therefore be described by one fixed, time independent transition matrix, A, from which the elements, $p_{i j}$, consist of the probability assigned to the transition of the grid from a defined state $i$ to a defined state $\mathrm{j}$ [6]-[8], [11]. Fig. 1 shows an example of a state space diagram of a simple DAMC, equation 1 shows its transition matrix A [8], [13].

$$
A=\left[\begin{array}{ccc}
(1-\lambda \Delta t) & \lambda \Delta t & 0 \\
0 & (1-\lambda \Delta t) & \lambda \Delta t \\
0 & 0 & 1
\end{array}\right]
$$

The DAMC can practically be simulated using the matrix multiplication method [6], [8], [11], given by equation 2 . With this method, the probabilities of the different states can be found after $n$ time steps, $\Delta \mathrm{t}$, using the transition matrix. Here, a row vector $\mathbf{P}_{0}$ is defined that consists of the initial state probabilities. Furthermore, row vector $\mathbf{P}_{n \Delta t}$ carries all the different state probabilities after $n$ time steps.

$$
\mathbf{P}_{n \Delta t}=\mathbf{P}_{0} A^{n}
$$

To be able to set up the transition matrix of an electrical grid, first a state space diagram (SSD) needs to be defined. To do this, several simplifications and assumptions are made. The most important ones are listed below:

- The protection system that needs to clear a faulty component in the grid has two levels. The first one, BV1, will work with a probability $p$ and comprises the opening of the two circuit breakers surrounding the faulty component. If BV1 fails, a second level of protection, BV2, works with a probability equal to one after its specified time delay. In this way, a larger part of the grid is usually switched off. This results in a final absorbing state with a larger inherent damage cost compared to a successful clearing by BV1.

- No double faults are allowed within the used simulation period. This assumption implies that whenever a fault occurs on a major grid component, no consecutive fault can occur in the grid on another major component until the grid is restored to its original state.

\section{Construction of the state space diagram}

The SSD contains all the defined states that can occur in the grid as well as the transitions between them [3], [12]. The determination of the different states in which the grid can reside is in this case mainly done based on a static analysis of the grid in its standard operating state. Fig. 2 shows the structure of the SSD. Here, the failures of the major grid components are taken into account together with the actions of the protection system (PS) and automatic switching actions (ASA) to recover the production which was switched off by a clearing command. Additionally, some dynamic actions are investigated and their results are implemented in the SSD as static states as the model doesn't integrate dynamic actions [7], [10].

The initial state $\left(P_{0}\right)$ of the SSD is the state in which the considered grid is working without any disturbances nor irregularities [13]. In this state, major faults can occur on the different described voltage levels with a probability of $\lambda_{i} \Delta \mathrm{t}$ [2]. Here, $\lambda_{i}$ is expressed in amount of failures of a component in 100 years. $\Delta \mathrm{t}$ must furthermore be small enough compared to the time scale of $\lambda_{i}$ as well as to the simulation time [6], [11]. After a major fault occurs in the grid, the protection system (PS) works in the next level of the SSD.

After the protection system actions, additional transitions of the grid can be initiated to restore the production which was switched off by the clearance of the fault. Two automatic switching actions (ASA) are considered in the analysis of the grid of BASF Antwerp:

- Switching Over (SO): The load is fed by one transformer. In case of problems, the load is taken over by another main transformer after this load experienced a short voltage interruption.

- Synchronizing Bus (SB): Several transformers are mutually connected through reactors with a common busbar: the synchronizing bus. In case of problems, the load that is fed by one transformer and the synchronizing bus is completely fed by the synchronizing bus without a voltage interruption (but with a short voltage dip).

The probabilities of these additional transitions, together with the actions of the protection system, are considered to be constant and time independent. This can be assumed as these actions take place immediately (seconds, milliseconds) 


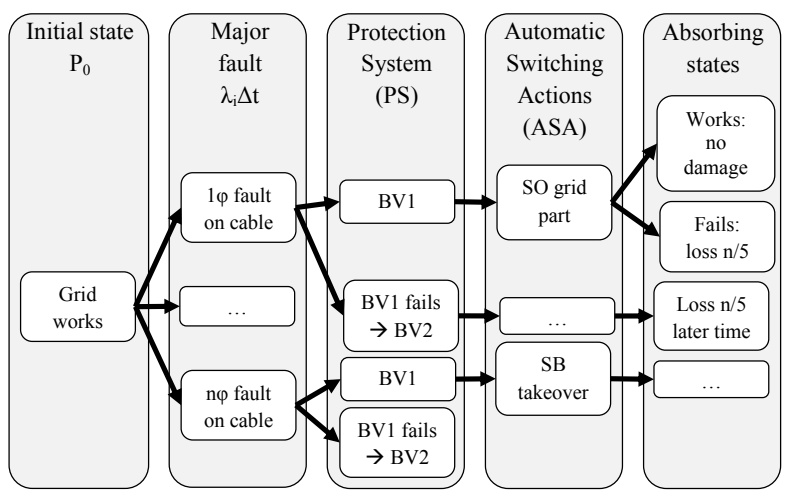

Fig. 2. A schematic representation of the structure of the state space diagram

compared to the time scale of the occurrences of the major faults in the grid, $\lambda_{i}$. Finally, after a couple of additional actions, the absorbing states of the grid are attained. Here, for example, the underlying production of one out of five or more sub-networks of the grid can be lost. The probabilities of these final states are used in the economic analysis (see section III-E) [11].

\section{Simulation}

This section provides a brief description of the two highest voltage levels of the grid of BASF Antwerp. This industrial grid is used to carry out two case studies with the proposed method of the DAMC combined with the matrix multiplication. In a last step, an economic analysis is performed with the results obtained from both case studies.

\section{A. Electrical grid of BASF Antwerp}

The grid of BASF Antwerp is powered by the Belgian grid operator Elia at the $150 \mathrm{kV}$ substation ZVN. From there on, two main transformers are fed directly and the other four main transformers are fed through substation BASF (see Fig. 3a). Each main transformer determines a sub-network. From the two transformers that are directly powered by substation ZVN, one is a spare transformer (hot standby), whereas the other one has its own consumers. The five main transformers feed five main busbars on the $35 \mathrm{kV}$ voltage level. These five main busbars are mutually connected through the SB and they all feed about $1 / 5$ of the total load. The SB is a busbar on the $35 \mathrm{kV}$ voltage level that has a connection with the five main busbars through reactors (see Fig. 3b).

\section{B. Case study I}

In a first step, a part of the grid of BASF Antwerp is simulated. The organization of the network is given in Fig. 4. Here, one main transformer is examined together with its most important connections. All these major connections can experience a single- or multiphase fault which leads to a total of 10 main faults that are introduced in the SSD of case study I. The effects of these different faults are analyzed with the Neplan software [14] and implemented in the SSD following the described structure (see Fig. 2).

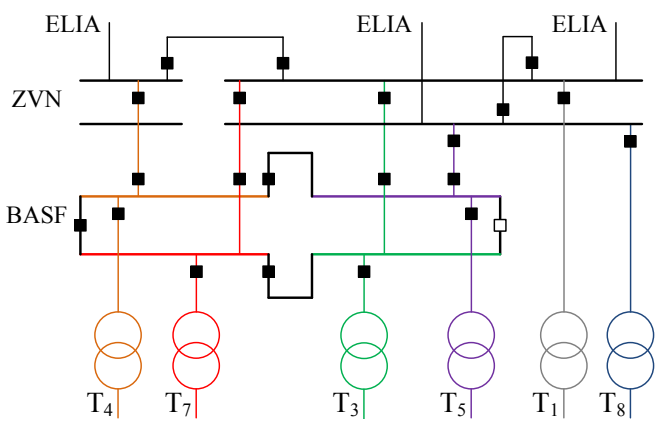

(a)

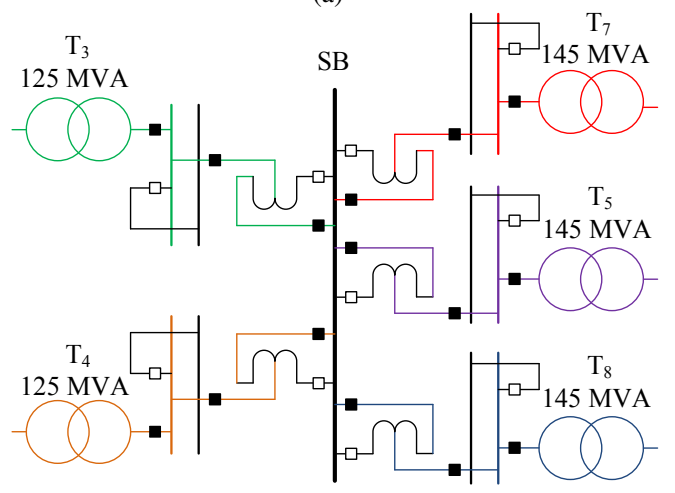

(b)

Fig. 3. A schematic representation of the electrical grid of BASF Antwerp: (a) the $150 \mathrm{kV}$ voltage level (b) the $35 \mathrm{kV}$ voltage level.

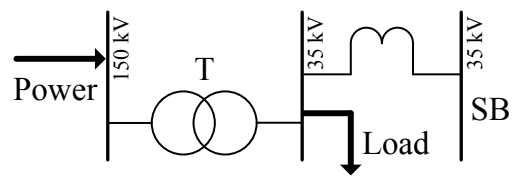

Fig. 4. A schematic representation of the part of the grid simulated in case study I

The resulting SSD consists out of 33 states. In this case, six absorbing final states are defined which are the damage states of the grid. One of these final states has no inherent damage cost towards the chemical production. This state, however, is a damage state compared to the initial state of the grid, as a faulty component has been switched off to attain it.

In a next step, probabilities must be assigned to all transitions in the SSD (see Section II). Thereafter, the matrix multiplication is used to simulate this network with a total simulation period of one year for $\Delta \mathrm{t}$ varying from 0.1 seconds up to 1 month. Here, the amount of time steps $n$ used in the simulation is respectively the amount of 0.1 seconds in one year up to the amount of months in one year. After every time step, the probability of the initial state together with the probabilities of the final damage states sums up to unity as the other states in the SSD are transient states [8], [11]. The obtained final state probabilities are plotted in Fig. 5 with respect to the different time steps. From the graph can be seen that the proposed method of the DAMC results in stable probability values after one year for a time step smaller than one day. For further simulations a $\Delta t$ of 1 minute is proposed 


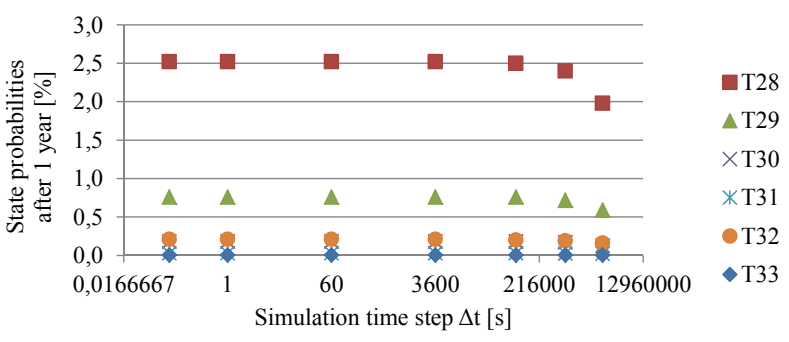

Fig. 5. The probabilities of the different absorbing states of the small grid as a function of $\Delta \mathrm{t}$, for a simulation period of 1 year

to operate well within this stable region.

The requirement of no double faults occuring within the simulation period must also be met. To obtain this, there is subsequently looked for a suitable simulation period which takes this requirement into account. Hereto, a first step towards repair is added to the model. The defined final state without any damage cost towards the production is linked back to the initial state through a constant repair rate, $\mu \Delta \mathrm{t}$ [8]. The MTTR is chosen to be 1 week, which results in a $\mu$ of 5200 times in 100 years. With this, the probability for this latter final state to succeed itself after each time step becomes $1-\mu \Delta \mathrm{t}$.

The simulation of the small grid with and without limited repair is subsequently carried out for the convergence towards steady-state. This convergence is shown for one random final state in Fig. 6a. Here can be seen that as long as both graphs coincide, the assumption of no double faults occuring within the simulation period is valid, as the probability of the state is then approximately equal for both cases. Thus, the requirement is valid within the linear coinciding part of both graphs. As the simulation period of one year falls within this region, a second fault can be neglected in this case study for this simulation period. As the considered grid in case study II consists out of more components, the assumption should be checked again for that case. For longer simulation periods, repair from every final state must be added to the simulation to obtain a higher level of accuracy of the model. In that way multiple faults are allowed within the simulation period.

\section{Case study II}

Now the applicability and stability of the proposed method are shown, it can be applied on a larger part of the grid of BASF Antwerp. This is again done according to the structure previously described (see Fig. 2), which subsequently leads to a SSD which consists out of 245 states and 30 final states.

First of all, the assumption of no double faults occuring within the simulation period must be checked for the large grid. Hereto, limited repair is installed in the large grid in the same way as was elaborated in case study I. The simulation is carried out for the large grid with and without limited repair for the convergence towards steady-state. Hereby, a time step of 1 minute is used. This evolution towards steady-state is shown in Fig. $6 \mathrm{~b}$ for a random final state. From the graph can be seen that the simulation period of one year falls beyond the linear part where both curves coincide. The simulation

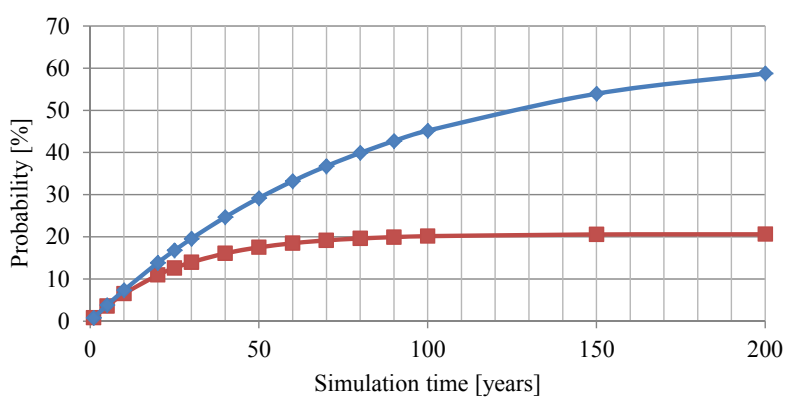

(a)

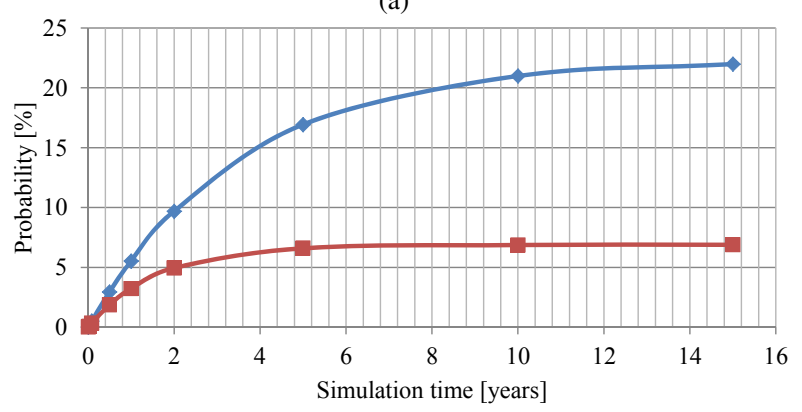

(b)

Fig. 6. The convergence towards steady state of a final state with (diamond) and without (square) limited repair of: (a) the small grid ( $\Delta$ t equal to 1 day) and of (b) the large grid ( $\Delta$ t equal to 1 minute).

periods that fall within this linear part provide approximately equal probabilities of the displayed state with and without limited repair. This result means that the proposed simulation period of one year must be reduced for the large grid for the probabilities to fall within the linear coinciding part of both graphs. Therefore, a simulation period of 1 week is chosen for further simulations of case study II. Due to the reduction in simulation period, $\Delta \mathrm{t}$ must be reduced as well to obtain stable state probabilities. As such, a time step is chosen that is small compared to the simulation period of 1 week, namely 1 second.

\section{Case study II: Impact of different grid configurations}

In a next step, the simulation of the large grid is performed for different configurations. First, Version I of the busbar insulations is proposed. Here, three of the $35 \mathrm{kV}$ sub-networks are Air Insulated Systems (AIS), likewise a part of substation ZVN. The other part of substation ZVN, substation BASF and the SB are unipolar Gas Insulated Systems (GIS), whereas two of the $35 \mathrm{kV}$ sub-networks are tripolar GIS. The various types of insulation have different failure rates. Here, AIS busbars are more likely to experience a single- or multiphase fault compared to GIS busbars because of protection against external influences of the latter and the age of the AIS system. Also, the unipolar GIS is more likely to experience a singlephase fault compared to a higher probability for a multiphase fault for the tripolar GIS. Version I is simulated for different grid configurations with a simulation period of 1 week and a $\Delta \mathrm{t}$ of 1 second. Each configuration excludes some actions or constructions in the grid like for example the SO or the SB. These simulations are performed to assess 
the added value and impact on the grid of these actions and grid constructions. In a next step, a second version of the busbar insulations is simulated. In this Version II three of the $35 \mathrm{kV}$ sub-networks are changed from AIS to unipolar GIS at their $35 \mathrm{kV}$ side. Every configuration results in different probabilities of the initial and final states. These probabilities are later used in the economic analysis (see subsection III-E) to determine the impact of the different configurations on the average damage cost that BASF Antwerp can expect after one year.

From the probabilities of the final states, the weakest grid elements are successfully identified. Grid components and subnetworks are weakest mainly due to AIS at their $35 \mathrm{kV}$ and/or $150 \mathrm{kV}$ side. This means that the busbars are in direct contact with the environment, whereas GIS protects the busbars in a system with SF6 gas under pressure. This explains the higher final state probabilities of the damage states of the AIS sub-networks. Another factor which introduces a higher final state probability of a certain sub-network is the length of the connecting cables between busbars and transformers. The longer the cable, the higher the probability of a singleor multiphase fault to occur as the failure rate of cables is expressed per $\mathrm{km}$. The weakest final states all result from failures in one particular sub-network. This is an expected result as this sub-network has AIS on both its $35 \mathrm{kV}$ and $150 \mathrm{kV}$ side. Moreover, it has the longest primary transformer cable of all sub-networks which implies the highest failure rate.

Taking into account the simulation results of Version II, the failure rates coupled to the different insulation forms are one of the major factors that influence the probabilities of the final states. The lower the probability of a single- or multiphase fault on a busbar, the lower the final state probabilities of damage states related to that sub-network. This leads to a lower expected overall damage cost.

\section{E. Economic analysis}

With the probabilities obtained from the different simulations of case study II, an economic analysis can be performed by linking a damage cost to each final state due to the loss of production associated with it [2], [12]. The obtained final state probabilities are each multiplied with their respective damage cost and are summed for each configuration. In this way, the average damage cost for BASF Antwerp to expect after a simulation period of one week is estimated. The expected average damage cost after one year can be approximated by multiplying this average damage cost with the amount of weeks in one year. These average damage costs for the different configurations are subsequently compared with each other. Through this more objective basis, some grid investments of BASF Antwerp could be justified. Also, it is shown that additional grid calculations are needed to assess the impact and the likeliness of some final states, as the resulting average damage cost is highly dependent on the accuracy of both the implemented transition probabilities as well as the damage costs associated with the final states. As such, the results of the performed analysis are only valid for the selected grid configuration and the used transition probabilities.

\section{CONCLUSIONS}

This paper proposes a model to obtain a more objective basis to evaluate both the reliability and the average cost of production loss due to faults in an industrial electrical grid. The results of the performed simulations in case studies I and II show that the proposed method of the discrete absorbing Markov chain together with the matrix multiplication is promising. Nevertheless, some minor drawbacks are identified. Firstly, the state space diagram tends to become quite complex and detailed for large grids. Secondly, the results of both the simulation and the economic analysis depend on the accuracy of the determined failure- and repair rates of the grid components as well as the accuracy of the damage costs assigned to the defined final states. As such, to improve the accuracy of the model, correct data are needed regarding failure- and repair rates as well as damage costs.

The merits of the proposed technique are illustrated through two case studies in which parts of the grid of BASF Antwerp are simulated. From these case studies, both the weakest parts of the grid of BASF Antwerp could be indentified as well as the average cost of production loss after one year. The discrete absorbing Markov chain technique is shown to have a lot of potential regarding the expansion to lower voltage levels in the grid, repair and double faults. These expansions will improve the accuracy of the model in further simulations.

\section{REFERENCES}

[1] R. Billinton and R. Allan, "Concepts of power system reliability evaluation," International Journal of Electrical Power \& Energy Systems, vol. 10, no. 3, pp. 139-141, 1988.

[2] _ - "Basic power system reliability concepts," Reliability Engineering \& System Safety, vol. 27, no. 3, pp. 365-384, 1990.

[3] R. Billinton and P. Kuruganty, "A probabilistic assessment of transient stability," International Journal of Electrical Power \& Energy Systems, vol. 2, no. 2, pp. 115-119, 1980.

[4] S. Verlinden, G. Deconinck, and B. Coupé, "Hybrid reliability model for nuclear reactor safety system," Reliability Engineering \& System Safety, vol. 101, pp. 35-47, May 2012.

[5] C. Wouters, "Betrouwbaarheidsanalyse van netconcepten - Een evaluatie van het elektrisch net van BASF Antwerpen," MSc thesis, K.U.Leuven, ESAT-ELECTA, 2012.

[6] R. Billinton and R. Allan, Reliability Evaluation of Engineering Systems: Concepts and Techniques. Pitman Publishing (USA), 1983.

[7] - Reliability evaluations of Power Systems. Pitman Publishing (USA), 1984.

[8] B. Johnson, Design and Analysis of FT Digital Systems. AddisonWesley (out-of-print), 1989.

[9] S. Logacio, "Electrical installation dependability studies," Cahier Technique Merlin Gerin, 1997, E/CT 184.

[10] A. Chowdhury and D. Koval, Power distribution system reliability: practical methods and applications. Wiley and IEEE press (USA), 2009.

[11] D. Logofet and E. Lesnaya, "The mathematics of markov models: what Markov chains can really predict in forest successions," Ecological modelling, vol. 126, no. 2-3, pp. 285-298, Feb. 2000.

[12] D. Elmakias, New computational methods in power system reliability. Springer (Berlin, Germany), 2008.

[13] R. Billinton and K. Bollinger, "Transmission system reliability evaluation using markov processes," IEEE Transactions on Power Apparatus and Systems, vol. PAS-87, no. 2, pp. 583-547, Feb. 1968.

[14] BCP Busarello + Cott + Partner AG, "Neplan." [Online]. Available: http://www.neplan.ch/ 